\title{
Testing the magnitude of correlations across experimental conditions
}

Simone Di Plinio ${ }^{1 *}$

${ }^{1}$ Department of Neuroscience Imaging and Clinical Sciences, “G. D'Annunzio" University of Chieti-Pescara, Chieti 66100, Italy;

* Corresponding author: Simone Di Plinio, Department of Neuroscience Imaging and Clinical Sciences, “G. D'Annunzio" University of Chieti-Pescara, Chieti 66100, Italy. simonediplinio@yahoo.it

Keywords: correlation, bootstrap, effect size, p value, mixed-effects.

Running head: Testing correlation across experimental conditions

\section{Conflict of Interest: The author declares he has no conflict of}

\section{interest.}

Ethical approval: This article is a simulation study and does not contain any studies with human participants or animals performed by any of the author.

Informed consent: Since the article is a simulation study, no informed consent was required. 


\begin{abstract}
Comparisons between correlation coefficients are used to investigate data across multiple research fields, as they allow investigators to determine different degrees of correlation to independent variables. Such differences may be small, even with adequate sample size, but still scientifically relevant. To date, although much effort has gone into developing methods for estimating differences across correlation coefficients, adequate tools for variable sample sizes and correlational strengths have yet to be tested. The present study evaluated four different methods for detecting the difference between two correlations and tested the adequacy of each method using simulations with multiple data structures. These methods were Cohen's $q$, Fisher's method, linear mixed-effects models (LMEM), and an ad-hoc developed procedure that integrates bootstrap effect size estimation. Results showed that Fisher's method and the LMEM tended to reject the null hypothesis even in the presence of relevant differences between correlations and that Cohen's method was not sensitive to data structure. Bootstrap followed by effect size estimation resulted in a favorable, unbiased compromise for estimating quantitative differences between statistical associations and producing outputs that could be easily compared across studies.
\end{abstract}




\section{Introduction}

Comparing statistics is a frequent point of contention among researchers. The need to compare correlations is common and requires a specific assay to determine whether a continuous variable, often called covariate, has a different degree of correlation between two sets of data. Examples of fields in need of such an assay include cognitive psychology (e.g., correlation between the degree of task automation and behavioral performance in extroverted vs. introverted individuals), social psychology (e.g., correlation between social exclusion and job satisfaction in men vs. women), and cognitive neuroscience (e.g., correlation between brain activity and behavioral performance under negative vs. positive emotional stimulation). Calculated differences may be small, even with the recommended sample size, but can still be scientifically relevant (Ellis, 2010). Furthermore, modern science is gradually moving away from $p$ centric data interpretation towards effect-size-oriented approaches (Kelley \& Preacher, 2012; Sullivan \& Feinn, 2012). As such, reporting only $r$ and $p$ values and binarizing result interpretations as either significant or non-significant, depending on an (eventually corrected) threshold of $p<0.05$, has become outdated (Nichols et al., 2017; Ioannidis, 2019).

The existence of a statistical association cannot be relied upon to evaluate whether the strength of the relation between two variables will always be the same. In a withingroup design, one of the correlation coefficients between the outcome (DV) and the covariate (IV) may be more diminished in a specific experimental condition $B$ than in another condition A. Alternatively, in a between-group design, the treatment group 
may show a weaker correlation between the outcome and the covariate than in the control group, or vice versa. These context-dependent or group-dependent effects on the extent of the correlation between two variables should be investigated using the most suited methods so that scientists from different disciplines can assess the most fitting comparison between the two correlations. Of note, given that null hypotheses are always false when evaluated with large datasets (Cohen, 1990), and that both small samples and large samples can convey useful information (Lindquist et al., 2012;

Friston, 2013), the effect of a correlational change should be investigated not only with various correlation values, but also various sample sizes. However, even though much effort has been invested into developing methodologies that can estimate differences across correlation coefficients, studies investigating this problem using a comprehensive approach and variable sample sizes have yet to be published. Several strategies have been developed to estimate differences between correlations. The simplest method was proposed by Jacob Cohen (Cohen, 1988), and estimated an effect size as the difference between two Fisher-transformed correlations. Fisher's method (Fisher, 1921) also accounts for sample size, and calculates the probability that two correlations will be different given their strength and the number of samples in the two groups. Whereas Cohen's and Fisher's methods rely exclusively on $r$ values, ignoring the initial data structure, analysis of covariance (ANCOVA) and linear mixedeffects models (LMEM) retain this information. ANCOVA and LMEMs have been widely adopted for analyzing data in cognitive neuroscience experiments, wherein the parameters observed are affected by multiple factors (Garrett et al., 2010; Zilles \& Amunts, 2013). 
In a recent neuroimaging study, a bootstrap approach followed by calculation of the effect size was used to detect changes in correlations between neurophysiological parameters and behavioral performance across experimental conditions (Di Plinio et al., 2018). This assay allowed testing of the hypothesis that the association between functional connectivity across brain regions and behavioral performance (Hampson et al., 2010) was weakened by negative emotional stimulation. Finally, structural equation modelling has also been proposed for testing independent or dependent correlational hypotheses (Cheung \& Chan, 2004). This method is grounded in confirmatory factor analysis and is useful when data includes both dependent and independent measures. However, this approach may be ill-suited to small sample sizes and is more appropriate for meta-analytic designs (Cheung \& Chuan, 2005; Cheung, 2014).

In this paper, four different approaches are examined to evaluate their power to detect an effect. The term effect in this study refers to "a change in the correlation between two conditions or two groups." Although $p$-values are undergoing a theoretical revision by the scientific community, they still provide a universally recognized statistic (Greenland, 2019; Goodman, 2019). As such, both $p$-values and effect estimates are reported for each method. The methods examined in this study are Cohen's' $q$, Fisher's method, LMEM, and bootstrap with effect size estimation. A series of simulations were implemented so that the four methods could be tested in an environment wherein correlational strength and sample size vary from one cycle to the next. Method performances are then discussed. 


\section{Materials and Methods}

\subsection{Simulation parameters}

In the simulations used in the present study, values of the first correlation coefficient, $r_{1}$, occurred in the interval $[-0.50 .5]$ in steps of 0.01 . For each value of $r_{1}$, the second correlation value $r_{2}$ occurred in the interval $\left[\left(r_{1}-0.5\right)\left(r_{1}+0.5\right)\right]$ in steps of 0.01 . For each cycle, $N$ samples were simulated for each condition. As such, $N$ will be the number of samples considered. $N$ varied from 10 to 180 , in steps of 2 . As our focus was on the comparison of repeated measures (within-subject design without missing data), the number of samples for each condition was set to be equal; that is, $N=n_{1}=n_{2}$. Correlation strengths and sample sizes were chosen in accordance with cognitive psychology- and neuroscience-like scenarios, but results can be extended to other research fields such as medicine and social psychology. Data were processed using MATLAB 9.2 (The Math Works Inc., Natick, MA). Statistics obtained for each method were averaged across values of $r_{1}$ and zero-centered. Each plot shows the average statistics (e.g., $p$-value, effect size) with respect to increasing values of $r_{2}$ and $N$, and across values of $r_{1}$

For LMEM and bootstrap models, sample data and covariate values were randomly generated and normally distributed, using the MatLab function randn. This led to the creation of two distributions for the dependent variable (DV), namely $\mathbf{X}_{\mathbf{1}}$ and $\mathbf{X}_{\mathbf{2}}$, with a mean $\mu_{1}=\mu_{2}=0$ and a standard deviation $\sigma_{1}=\sigma_{2}=1$. Independent variable (IV) and $N$ covariate values were simulated for each condition and computed to yield the desired 
value of correlation with the DV. The covariates $\gamma_{1}$ and $\gamma_{2}$ were obtained as follows:

$\boldsymbol{\gamma}_{i}=\sigma_{\gamma}\left(r_{i} * \chi_{i}+\sqrt{1-r_{i}^{2}} * \boldsymbol{\Psi}_{i}\right)+\mu_{\gamma}$

where $\mu_{\nu}$ and $\sigma_{\nu}$ are the desired mean and standard deviation of the covariate (with $\mu_{\nu}$ $=0$ and $\left.\sigma_{\gamma}=1\right)$, respectively, while $\psi$ is a pseudo-random set of $N$ normally distributed values. This procedure was based on the Cholesky decomposition, which is commonly used in Monte-Carlo simulations of multiple correlated variables (Press et al., 1992).

\subsection{Methods}

\subsubsection{Cohen's $q$}

Cohen proposed a simple method for interpreting the difference between two correlations (Cohen, 1988). Initially, to reduce skewness (asymmetry derived from the definition of $r_{i}$ in the interval [-1 1]), $r$ values were transformed to $z$ values via the Fisher procedure:

$z_{i}=(0.5) \log \left(\frac{1+r_{i}}{1-r_{i}}\right)$

Then, the absolute value of the difference between the two $z$ values was computed, such that $q=\left|z_{1}-z_{2}\right|$. The value $q$ is the estimate of the effect size. The following intervals were proposed by Cohen to interpret these values: $q<0.1$, no effect; $0.1 \leq q<$ 0.3 , small effect; $0.3 \leq q<0.5$, medium effect; $q \geq 0.5$, large effect. Since no $p$-value is associated with Cohen's method, the only statistic reported is the effect size $q$. 


\subsubsection{Fisher's method}

Fisher's method (Fisher, 1921) is used to calculate the probability of two correlations being different, given the differences between $r$ values and the size of the two samples. The null hypothesis is that the correlation between $\mathbf{X}_{\mathbf{1}}$ and $\boldsymbol{\gamma}_{\mathbf{1}}$ will be the same as the correlation between $\mathbf{X}_{2}$ and $\boldsymbol{\gamma}_{2}$ for sample sizes $n_{1}$ and $n_{2}$. Correlation values $r_{1}$ and $r_{2}$ are converted to $z$ values as described in Equation 2. The test statistic $t$ is then calculated:

$t=\frac{\left(z_{1}-z_{2}\right)}{\sqrt{1 /\left(\mathrm{n}_{1}-3\right)+1 /\left(\mathrm{n}_{2}-3\right)}}$

Finally, using the cumulative distribution function of $t$ in a standard distribution with mean $\mu_{\mathrm{t}}=0$ and standard deviation $\sigma_{\mathrm{t}}=1$, the $p$-value is calculated to assess whether the null hypothesis can be trusted or not. Statistics reported for Fisher's method are the $p$-value and $t$-statistic.

\subsubsection{Linear mixed-effects model}

Linear mixed-effect models (LMEM) were applied in the form [DV IV*condition $+(1 \mid$ subject $)]$ and thus included a fixed effect (the experimental condition), a continuous effect (the covariate IV), and a random intercept at the subject level (1| subject) to account for inter-individual variability. This type of model is applied frequently in psychology and neuroscience. At each cycle, a model was fitted using the MatLab function fit/me and the $p$-value and $\beta$ statistics for the interaction between the experimental condition and the covariate were extracted. 
Among applicable generalized linear models, the choice of the LMEM over, say, ANCOVA is due to the former's increased flexibility and sensitivity (Schneider et al., 2015; Brysbaert \& Stevens, 2018). Of note, as the aim of the present work is to investigate the power to predict an effect, corrections for multiple comparisons were unnecessary. Furthermore, since within-subject variability at the interaction level (subject : covariate) was not simulated, the introduction of random slopes was not necessary for the purposes of the study.

\subsubsection{Bootstrap method and effect size estimation}

The bootstrap method is a resampling technique often used to estimate confidence intervals and allows one to approximate the sampling distribution of a statistic (Efron \& Tibshirani, 1986). In this study, we used a univariate, bias-corrected, accelerated bootstrap with replacement (Efron, 1987) to sample the correlation value $r_{i}$. A sampling distribution was obtained by resampling the original data $k$ times and obtaining $k$ samples with sizes equal to the starting sample $\left(N_{k}=N\right)$; that is, $k$ is the number of bootstrap cycles. A similar sampling approach has been described for correlations (see

Olkin \& Finn, 1990, 1995); however, the method presented here implements a biascorrected bootstrap procedure that can accommodate small sample sizes and outliers and includes an effect size estimation statistic.

Each cycle was bootstrapped by estimating the correlation between the DV and covariate for each condition. Individually bootstrapping each correlation allows the estimation of the bootstrapped effect size for each condition, which may be useful for descriptive purposes. After $k$ bootstrap cycles, two distributions of correlations were 
obtained for each condition and transformed into z-values. Each distribution possessed an associated mean and standard deviation $\left(\mu_{z 1}, \sigma_{z 1} ; \mu_{z 2}, \sigma_{z 2}\right)$. These distributions were then used for analyses. For each cycle (i.e., for each pair of $r_{2}$ and $N$ values), the difference between the two z-distributions was represented as indicated in Equations 4 and 5, with the effect size estimated using Cohen's $d$ (Lipsey \& Wilson, 2001; Ellis, 2010):

$\mathrm{ES}_{12}=d=\frac{\mu_{z 1}-\mu_{z 2}}{\sigma_{\text {pooled }}}$

where

$\sigma_{\text {pooled }}=\sqrt{\frac{\left(\mathrm{n}_{1}-1\right) \sigma_{z 1}^{2}+\left(\mathrm{n}_{2}-1\right) \sigma_{\mathrm{z} 2}^{2}}{\mathrm{n}_{1}+\mathrm{n}_{2}-2}}$

A Z-test was performed comparing the distribution obtained by subtracting the two bootstrapped correlation distributions against a zero-centered distribution, to prevent biases caused by large sample sizes in estimating a $p$-value. Effect sizes (ES) can also be interpreted in terms of the percentage of nonoverlap of the first group's scores with those of the second group (Cohen, 1988). For example, ESs of 0.0, 0.8, and 1.7 indicate that the distribution of scores for the first group overlapped with the distribution of scores for the second group with $0,47.45$, and $75.4 \%$ of nonoverlap, respectively.

\section{Results}

Results of the simulations are reported in Figures 1-4. In these figures, the $x$ - and $y$-axes represent sample size and correlation difference, respectively, between $r_{1}$ and $r_{2}$. Values are averaged across different levels of $r_{1}$ tested. Total time to run the simulation 
was approximately 75 hours on a personal computer with eight $2.40 \mathrm{GHz}$ core processors.

Figure 1 shows the results of Cohen's $q$ method, which returns an estimate of the effect $(q)$ independent from the sample size $N$, reflecting only the difference between correlations. Empirically, small, medium, and large effects are defined based upon the magnitude of $q$. The interpretation of the result needs to be contextualized, however; a large difference between two correlations in a small sample $(N<10)$ is not systematically trustworthy. Conversely, a small effect might still be an important one (Rosnow \& Rosenthal, 1989).

Figure 2 show $p$-values and $t$-statistics obtained using Fisher's method. The test did not return significant values (Figure 1b, red) for relatively small sample sizes, even those with large differences between $r_{1}$ and $r_{2}(\Delta r=0.4)$. For larger samples $(N=40)$, however, this method accepted the null hypothesis as indicated by $\Delta r=0.15$. In the formula used for the $t$-statistic, sample size increases cause the test statistics to increase logarithmically, with the $p$-values showing a logarithmic decrease. Results of the mixed methods analysis (LMEM) are reported in Figure 3. Like ANCOVA, mixed models are likely to accept the null hypothesis even in the presence of a large difference between $r_{1}$ and $r_{2}$, as it interprets this difference as not being significant. This happens even with adequate sample sizes, such as an $N$ of 40 , by neuroscience standards. An advantage of LMEMs, however, is that the means and standard deviations of the original data are retained. Results thereby depend upon how the data points are distributed, and graphs tend to be more scattered than those described in previous paragraphs. 
The $p$-value and effect size $d$ obtained with bootstrapping simulations are reported in Figure 4. Like in previous methods, $N$ and $r_{2}$ vary while $r_{1}$ is fixed. The number $(k)$ of random samplings was set to $200(k=200)$. Equivalent results were obtained for pilot simulations with $k=500$ and $k=1,000$; however, as these simulations included a reduced number of cycles for computational purposes, their results are not included here. As for Cohen's method, the bootstrap approach provides an effect size estimation but not a $p$-value. Moreover, the procedure is sensitive to variability in the data, as indicated by the smoothness of the colors.

A post-hoc comparison among $p$-values gathered using Fisher, LMEM, and bootstrap methods is reported in Figure 5 . The bootstrap method was quite unaffected by the sample size, accepting more frequently the null hypothesis only with very small samples (e.g., $N<20$ ). Conversely, both Fisher and LMEM accepted the null hypothesis even with large differences between correlations, whereas the inverse bias was observed with large samples.

Since it may be pointed out that the results described until now may be based on averages across values of $r_{1}$, an example with a specific value of $r_{1}\left(r_{1}=0.30\right)$ is illustrated in Figure 6. On the one hand, these results confirm the observations made until this point: Cohen's method ignores sample size; Cohen's and Fisher's methods do not account for variability in the data structure; Fisher's method and LMEM tend to accept the null hypothesis even with large differences in the correlational strength. However, the presence of overestimated effect sizes (see for example some points with $\mathrm{N}<20$ in Figure $6 \mathrm{f}$ for $\mathrm{LMEM}$ ) shows that the variability in the data structure may endanger the estimation of fixed-effects statistics in mixed-models (Faraway, 2006) 
probably falling on Simpson's paradoxes (Good \& Mittal, 1987). The bootstrap procedure still accounted for variability in the data, but these paradoxical cases were not observed. This probably happened because the bootstrap procedure limits the pitfalls of classical inferences methods (Killeen, 2005).

\section{Discussion}

The present study compared vintage and modern statistical methods used to evaluate differences across correlations in the fields of psychology, medicine, and related disciplines.

A direct comparison between correlation coefficients as provided by Cohen's method can be useful, given that the calculation only requires the two correlation values (Cohen, 1988). However, Cohen's method estimates the effect magnitude irrespectively of sample size. Conversely, the effect estimated by Fisher's method increases with increasing sample size. Although these two methods can be useful when the original data structure is unavailable, neither Fisher's nor Cohen's method consider potential variability in the data. As such, the effects estimated with these methods should be carefully contextualized and interpreted.

It has been suggested that LMEMs may generate $p$-values that are too small, possibly overestimating the importance of a given effect (Faraway, 2006). The primary purpose of the LMEM is to use data from a continuous variable to estimate differences between levels of a fixed factor, including repeated-measures or longitudinal scenarios (Robinson, 1991). Given the results presented here, LMEM (and other generalized 
linear models, like the ANCOVA) may be useful for explorative purposes but may not be the best choice if the aim is to test the difference between the two correlations. In fact, the presence of overestimated effect sizes for LMEM suggested possible miscalculation on fixed-effects coefficients in mixed-models (Faraway, 2006; Good \& Mittal, 1987). The bootstrap procedure followed by a test to determine effect size accurately estimated the difference between the strength of the two correlations. Key features of this method are that it considers the sample size and the variability of the initial data, returns a descriptive measure of the difference between the two correlations, and provides a $p$-value not biased by too small or too large sample, and not affected by the pitfalls of classical inferences methods (Killeen, 2005).

Although Fisher, LMEM, and bootstrap methods returned a $p$-value, which is traditionally used to assess the presence or absence of an effect, the debate about how and even if these statistics should be used persists (Goodman, 2019). Certain seminal cognitive neuroimaging studies dealt with relatively small samples (Lindquist et al., 2012; Friston, 2013) due to logistical constraints, and such samples may not be large enough to detect significant differences using linear regression (LMEM). Recently, $p$ values have been shown to be misleading measures of the strength of the evidence against the null hypothesis (Berger \& Selke, 1987; Hupé, 2017); also, they do not directly provide an index of effect magnitude (Sullivan \& Feinn, 2012). The fact that the bootstrap approach provided a descriptive effect size which was close to the effect estimated by mixed-models underlines its effectiveness and is in line with the trend towards $p$-independent assays in psychology (Ellis, 2010; Kelley \& Preacher, 2012; Nichols et al., 2017). Furthermore, the implementation of a z-test provided an 
unbiased, universally accepted null-hypothesis statistic that may still be useful for $p$ generation researchers (loannidis, 2019; Greenland, 2019). Bootstrapping correlations have been discussed extensively in the literature, and researchers have noted that monotonic, transformation invariant procedures like the bootstrap are ill-suited to estimating confidence intervals or testing a null hypothesis (Lunneborg, 1985; Efron \& Tibshirani, 1986; Rasmussen, 1987; Strube, 1988; Efron, 1988; Olkin \& Finn, 1995). The procedure presented here and compared with other methods, however, represents a slightly new approach that combines bootstrap with effect size estimation and nullhypothesis testing.

\section{Conclusion}

The present study evaluated the efficacy of four different methods for investigating differences in correlations across experimental conditions. Bootstrapping followed by effect size estimation was the most successful, providing a statistic that accounted for both inter-individual and sample size variability in comparing correlation coefficients between experimental conditions. This method is easily implementable in MatLab through the bootes function made available online by the author at MathWorks. Although these findings have implications for researchers interested in comparing the magnitude of correlations between different experimental conditions, this study has two important limitations that must be acknowledged. First, the series of simulations implemented here only considered normally-distributed sample data with zero mean or unitary variance. Second, the bootstrap procedure presented here works well for 
within-subject analyses and may be applied without complications to those between subjects, but is not yet applicable to experimental designs including both between- and within-subject factors. Future studies should evaluate these and other methods in such alternative situations, to uncover other easily-implemented, bias-free tools for researchers in the fields of psychology, neuroscience, and medicine. 


\section{References}

Berger, J O \& Sellke, T. 1987. Testing a Point Null Hypothesis: The irreconcilability of $p$ values and evidence. Journal of the American Statistical Association, 82(397), 112122.

Brysbaert, M \& Stevens, M. 2018. Power analysis and effect size in mixed models: A tutorial. Journal of Cognition, 1(1), 9.

Buckner, R L, Andrews-Hanna, J R, Schacter, D L. 2008. The brain's default network: Anatomy, function, and relevance to disease. Annals of the New York Academy of Sciences, 1124, 1-38.

Cheung, M L \& Chan, W. 2004. Testing dependent correlation coefficients via structural equation modelling. Organizational Research Methods, 7(2), 206-223.

Cheung, M L \& Chan, W. 2005. Meta-analytic structural equation modelling: A twostage approach. Psychological Methods, 10(1), 40-64.

Cheung, M L. 2014. Fixed- and random-effects meta-analytic structural equation modeling: Examples and analyses in R. Behavior Research Methods, 46(1), 29-40.

Cohen, J. 1988. Statistical power analysis for the behavioral sciences. Lawrence Erlbaum associates, Second Edition.

Cohen, J. 1990. Things I have learned (so far). The American Psychologist, 45, 13041312.

Di Plinio, S, Ferri, F, Marzetti, L, Romani, G L, Northoff, G, Pizzella, V. 2018. Functional connections between activated and deactivated brain regions mediate emotional interference during externally-directed cognition. Human Brain Mapping, 39(9), 3597-3610.

Ellis, P D. 2010. The essential guide to effect sizes - Statistical power, Meta-analysis, and the interpretation of research results. Cambridge University press, ISBN: 9780511761676

Efron, B \& Tibshirani, R. 1986. Bootstrap methods for standard errors, confidence intervals, and other measures of statistical accuracy. Statistical Science, 1(1), 54-75.

Efron, B. 1987. Better bootstrap confidence intervals. Journal of the American Statistical Association, 82(397), 171-185.

Efron, B. 1988. Bootstrap confidence intervals: Good or bad? Psychological Bulletin, 104, 293-396.

Faraway, J J. 2006. Extending the Linear Model with R: Generalized Linear, Mixed Effects 
and Nonparametric Regression Models. Chapman \& Hall/CRC Taylor \& Francis Group, ISBN: 0-203-49228-5.

Fisher, R A. 1921. On the probable error of a coefficient of correlation deduced from a small sample. Metron, 1, 3-32.

Friston, K. 2013. Sample size and the fallacies of classical inference. Neurolmage, 1(81), 503-504.

Garrett, D D, Kovacevic, N, McIntosh, A R, Grady, C L. 2010. Blood oxygen leveldependent signal variability is more than just noise. Journal of Neuroscience, 30(14), 4914-4921. doi:10.1523/JNEUROSCI.5166-09.2010

Good, I J \& Mittal, Y. 1987. The amalgamation and geometry of two-by-two contingency tables. The Annals of Statistics, 15(2), 694-711.

Goodman, S N. 2019. Why is Getting Rid of P-Values So Hard? Musings on Science and Statistics. The American Statistician, 73, 26-30.

Greenland, S. 2019. Valid P-Values Behave Exactly as They Should: Some Misleading Criticisms of P-Values and Their Resolution With S-Values. The American Statistician, 73, 106-114.

Hampson, M, Driesen, N, Roth, J K, Gore, J C, Constable, R T. 2010. Functional connectivity between task-positive and task-negative brain areas and its relation to working memory performance. Magnetic Resonance Imaging, 28(8), 1051-1057.

Huang, A S, Klein, D N, Leung, H C. 2016. Load-related brain activation predicts spatial working memory performance in youth aged 9-12 and is associated with executive function at earlier ages. Developmental Cognitive Neuroscience, 17, 1-9.

Hupé, J. 2015. Statistical Inferences under the null hypothesis: common mistakes and pitfalls in neuroimaging studies. Frontiers in Neuroscience, 9(18), doi: 10.3389/fnins.2015.00018.

Ioannidis, J P A. 2019. What have we (not) learnt from millions of scientific papers with $p$ values? The American Statistician, 73, 20-25.

Kelley, K \& Preacher, K J. 2012. On effect size. Psychological Methods, 17(2), 13-152.

Killeen, P R. 2005. An Alternative to Null-Hypothesis Significance Tests. Psychological Science, 16(5), 345-353.

Lindquist, M A, Caffo, B, Crainiceanu, C. 2012. Ironing out the statistical wrinkles in "ten ironic rules". Neurolmage, 1(81), 499-502.

Lipsey, M W \& Wilson, D B. 2001. Practical Meta Analysis, volume 49. SAGE Publications. 
Lunneborg, C E. 1985. Estimating the correlation coefficient: Bootstrap and parametric approaches. Psychological Bulletin, 98, 209-215.

Nichols, T E et al. 2017. Best practices in data analysis and sharing in neuroimaging using MRI. Nature Neuroscience, 20(3), 299-303.

Olkin, I \& Finn, J D. 1990. Testing correlated correlations. Psychological Bulletin, 108(2), 330-333.

Olkin, I \& Finn, J D. 1995. Correlations redux. Psychological Bulletin, 118, 155-164.

Press, W H, Teukolsky, S A, Vetterling, W T, Flannery, B P. 1992. Numerical Recipes in C. The art of Scientific Computing, volume 55. Cambridge University Press. Cambridge, Second Edition, ISBN: 978-0521750332.

Rasmussen, J L. 1987. Estimating the correlation coefficient: Bootstrap and parametric approaches. Psychological Bulletin, 101, 136-139.

Robinson, G K. 1991. That BLUP is a Good Thing: The Estimation of Random Effects. Statistical Science, 6(1), 15-32- doi: 10-1214/ss/1177011926.

Rosnow, R L \& Rosenthal, R. 1989. Statistical procedures and the justification of knowledge in psychological science. American Psychologist, 44(10), 1276-1284.

Schneider, B A, Avivi-Reich, M, Mozuraitis, M. 2015. A cautionary note on the use of the Analysis of Covariance (ANCOVA) in classification designs with and without within-subject factors. Frontiers in Psychology, 6(474), 1-12.

Strube, M J. 1988. Bootstrap type I error rates for the correlation coefficient: An examination of alternative procedures. Psychological Bulletin, 104, 290-292.

Sullivan, G M \& Feinn, R. 2012. Using effect size - or Why the $P$ value is not enough. Journal of Graduate Medical Education, 3(3), 279-282.

Tabachnick, B \& Fidell, L S. 2012. Using Multivariate Statistics. Pearson, Sixth Edition, ISBN: 9789352861750.

Zilles, K \& Amunts, K. 2013. Individual variability is not noise. Trends in Cognitive Sciences, 17(4), 153-155. 


\section{FIGURES AND CAPTIONS}

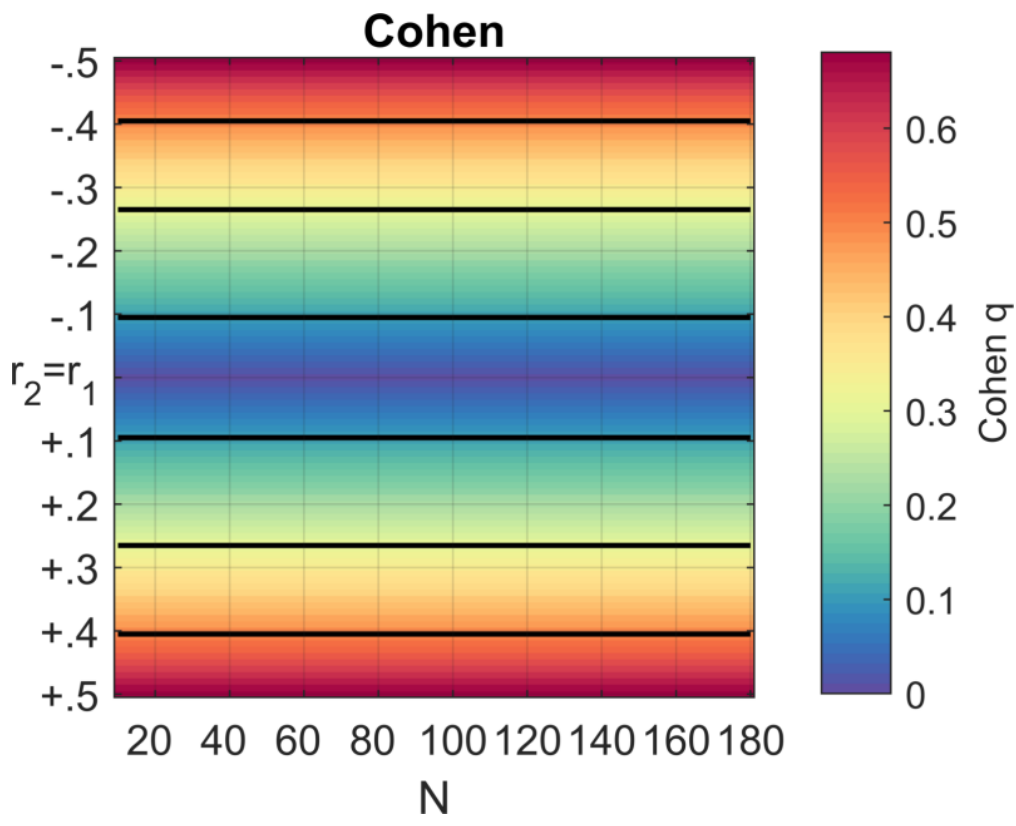

Figure 1. Results of the simulations using Cohen's $q$. Effect sizes are averaged across values of $r_{1}$. Black horizontal lines are used to separate the different levels of effect: small (blue), medium (cyan), large (green). Sample size is represented in the horizontal axis; values of $r_{2}$ with respect to $r_{1}$ are represented in the vertical axis. 

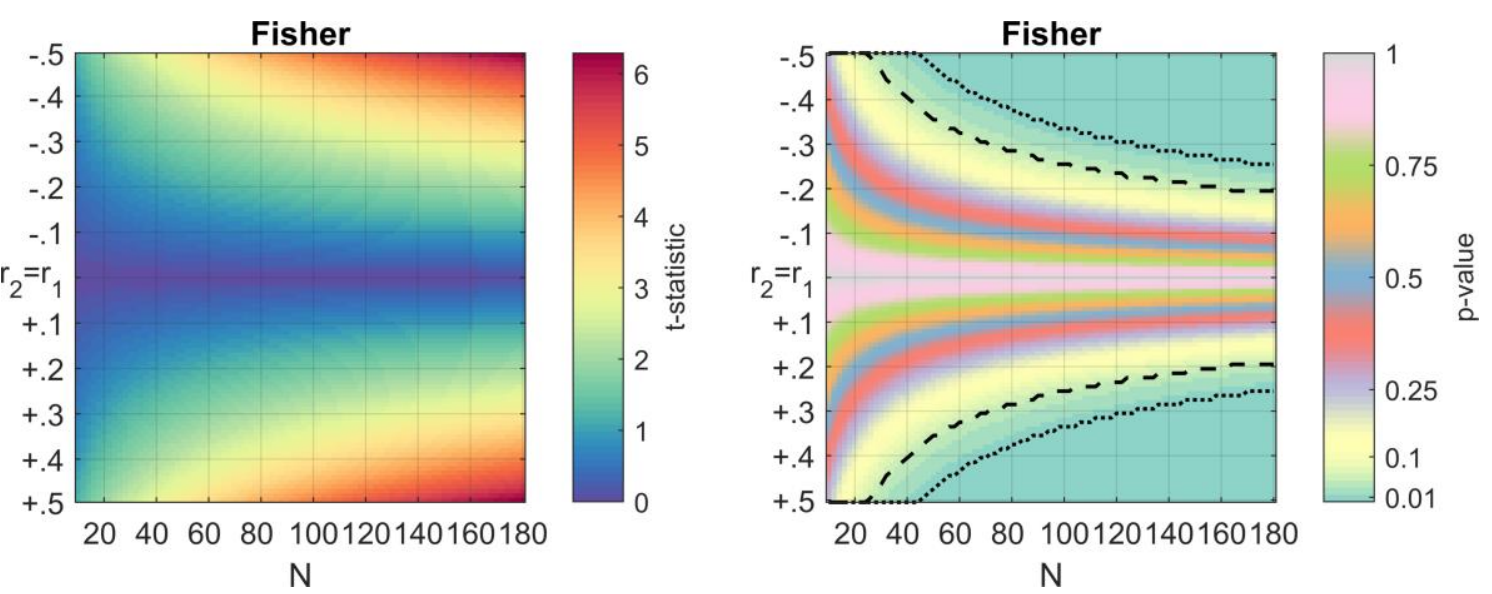

Figure 2. Results of the simulations using Fisher's method. Statistics are averaged across values of $r_{1}$. $T$-statistics are reported on the left panel, $p$-values on the right panel. Sample size is represented in the horizontal axis; values of $r_{2}$ with respect to $r_{1}$ are represented in the vertical axis. Dashed and dotted lines represent thresholds of $p<0.05$ and $p<0.01$, respectively. 

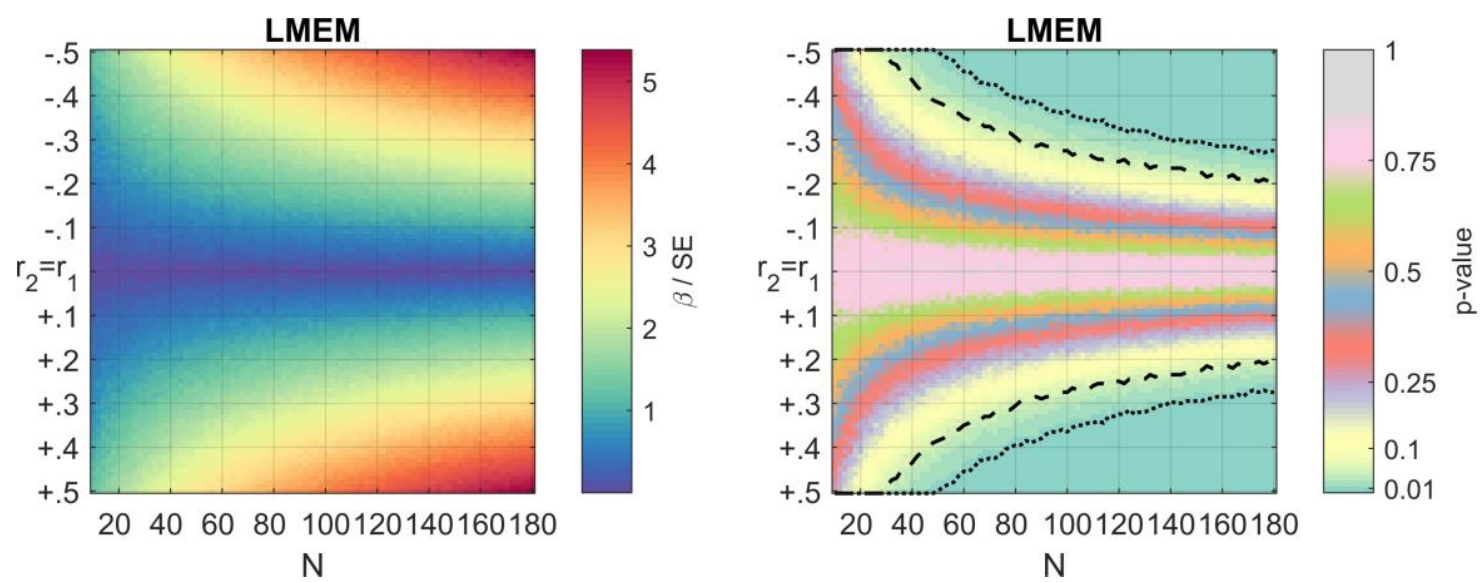

Figure 3. Results of the simulations using mixed-models (LMEM). Statistics are averaged across values of $r_{1}$. $\beta$ values for the interaction condition : covariate divided by their standard errors (SE) are reported on the left panel, corresponding $p$-values are reported on the right panel. Sample size is represented in the horizontal axis; values of $r_{2}$ with respect to $r_{1}$ are represented in the vertical axis. 

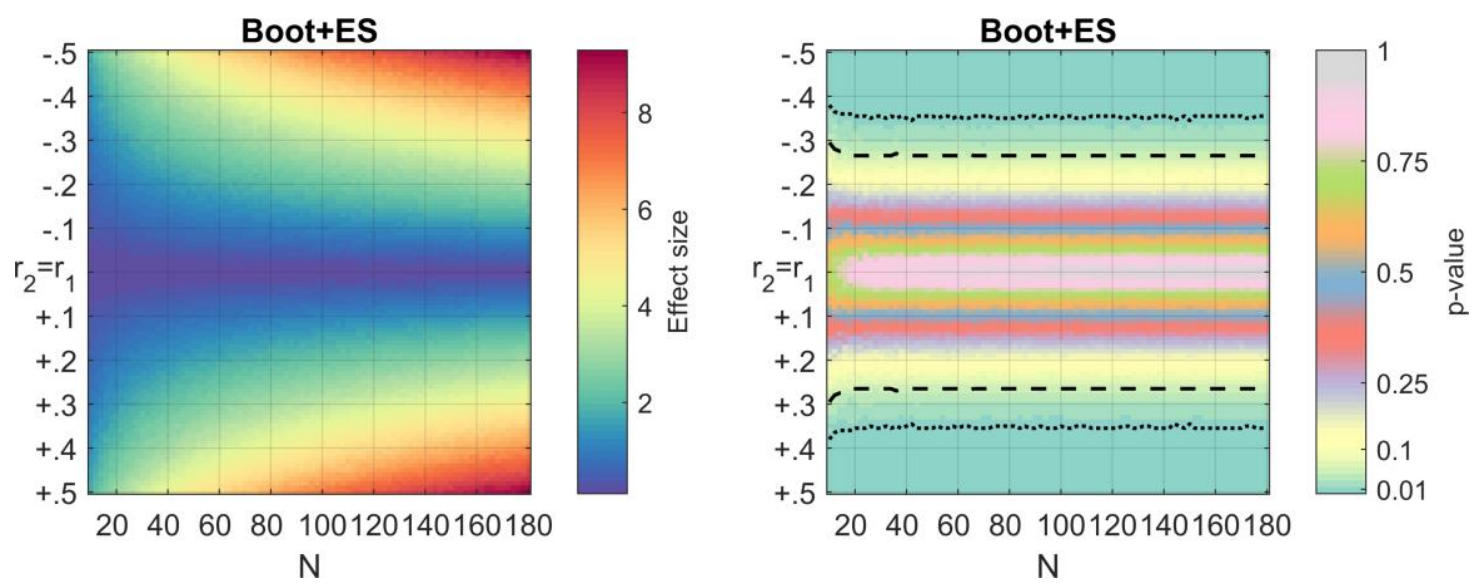

Figure 4. Results of the simulations using bootstrap (Boot+ES). Statistics are averaged across values of $r 1$. Effect sizes $(d)$ are reported on the left panel, corresponding $p$ values are reported on the right panel. Sample size is represented in the horizontal axis; values of $r_{2}$ with respect to $r_{1}$ are represented in the vertical axis. 


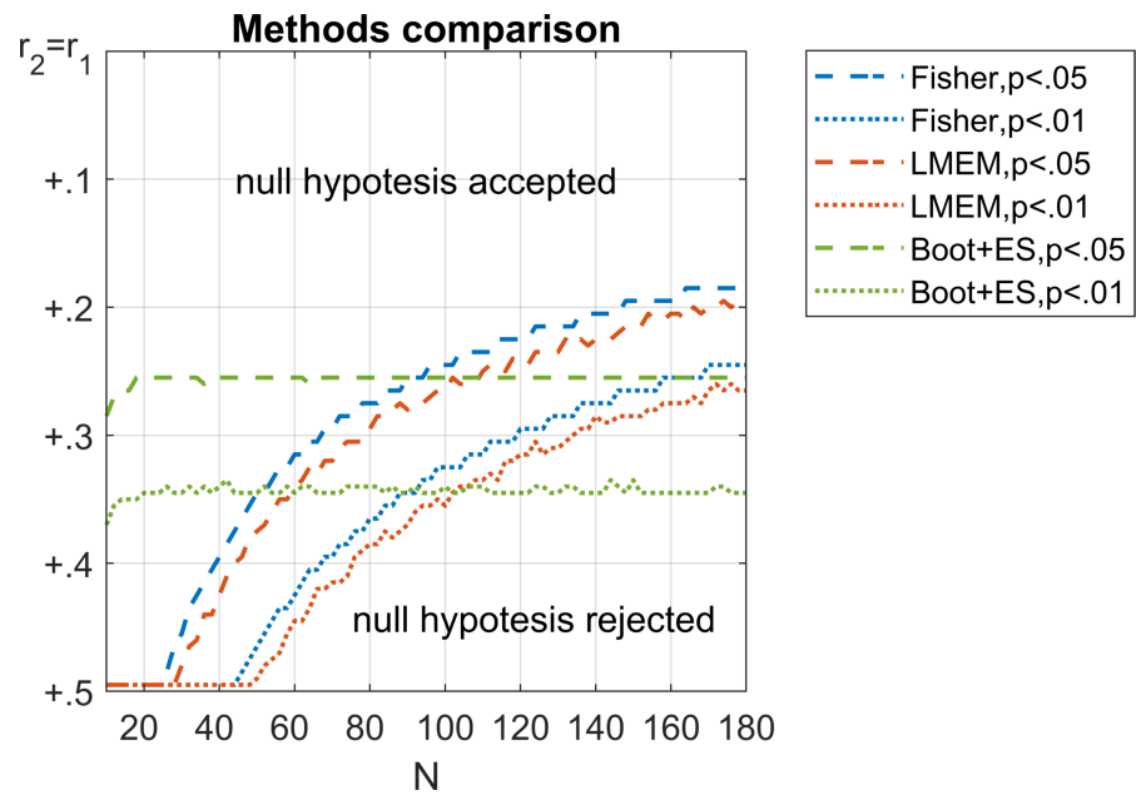

Figure 5. Comparison among Fisher (in blue), LMEM (in red), and bootstrap (in green) $p$-values. Thresholds of $p=0.05$ (dashed lines) and $p=0.01$ (dotted lines) are represented for each method. For graphical clarity, only the lower half of the values are reported here. 

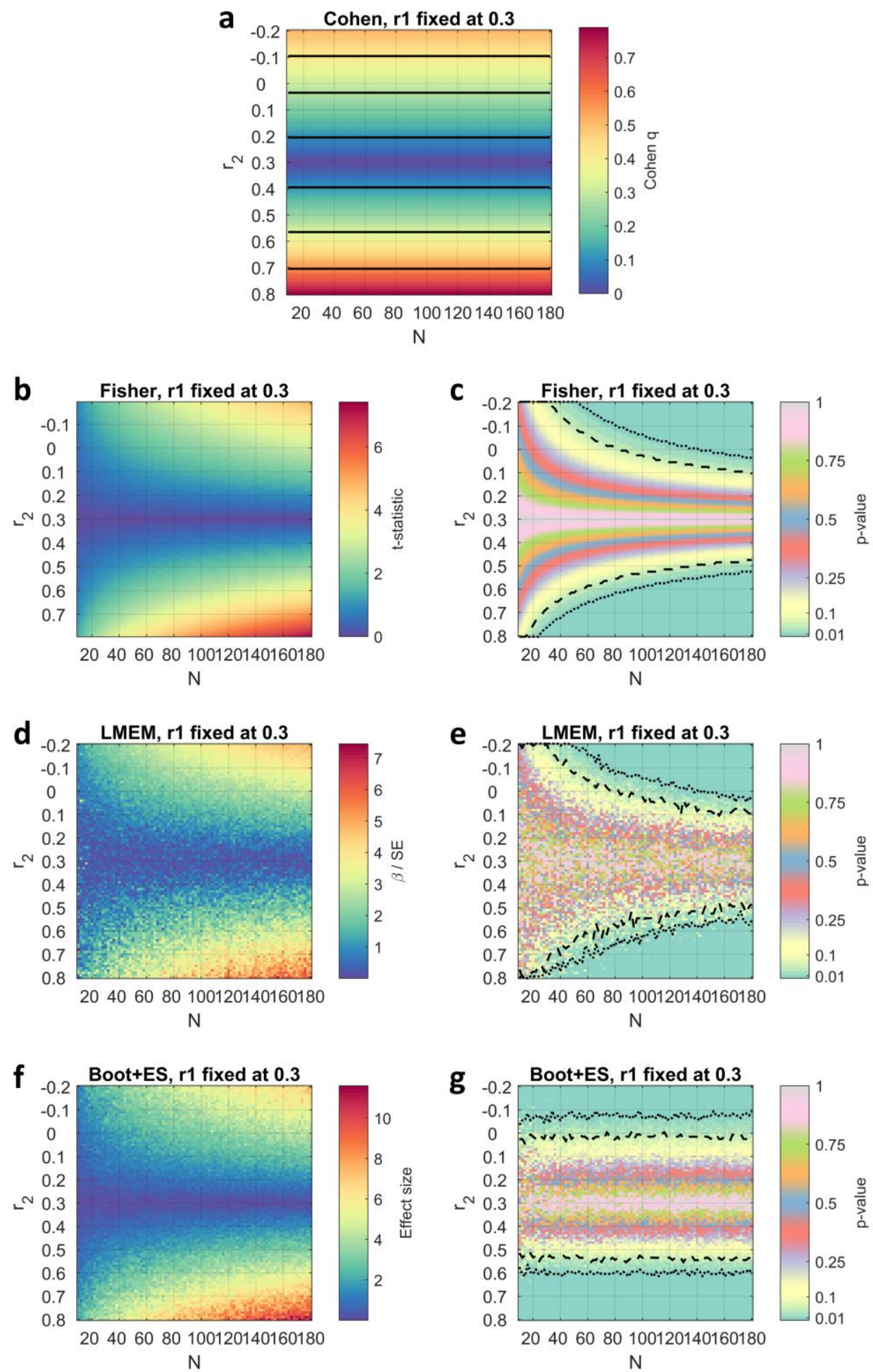

Figure 6. Results of the simulations using the four methods, with $r_{1}=0.30$. Dashed and dotted lines represent thresholds of $p<0.05$ and $p<0.01$, respectively. Sample size is represented in the horizontal axis; values of $r_{2}$ with respect to $r_{1}$ are represented in the vertical axis. (a) Cohen's method. (b-c) Fisher's method. (d-e) LMEM. (f-g) Bootstrap followed by effect size estimation. 